\title{
Apropiaciones, necesidades y derechos de la comunicación radiofónica en el norte de Argentina
}

\author{
Recibido: 19 de noviembre de 2015 \\ Aceptado: 01 de julio de 2016 \\ Publicado: 30 de noviembre de 2016
}

\author{
Emiliano Venier \\ evenier@unsa.edu.ar \\ Universidad Nacional de Salta (Argentina)
}

Resumen: Las prácticas que vinculan a las personas con los dispositivos comunicacionales pueden pensarse como respuestas a necesidades de los grupos sociales que promueven formas diversas de hacer, pensar y gestionar la comunicación. En esa articulación entre público, medios y políticas de comunicación se expresaría un modo de reconocer el derecho a la comunicación. Sin embargo, la demanda mediante la cual se expresa la necesidad de la comunicación como derecho no necesariamente agota la diversidad de formas posibles del derecho a la comunicación ni tampoco las maneras de atenderlas.

El trabajo propone ensayar los argumentos que sostienen la siguiente idea: si en el caso de la Argentina, las actuales políticas públicas de comunicación reconocen como fundamento los principios de pluralidad y democracia, y, además, es posible dar cuenta de una diversidad de modalidades de apropiación y uso de los medios en virtud de diversas necesidades. Entonces resulta fundamental para las políticas de comunicación en la diversidad reconocer la multiplicidad de necesidades simbólicas y de sus formas de satisfacerlas.

Palabras clave: Derecho a la comunicación, necesidades comunicacionales, apropiaciones y usos de la radio, comunicación y cultura.

Abstract: Practices that link people with communication devices can be thought of as responses to needs of social groups that give rise to different ways of doing, thinking and managing communication. In this articulation between public, media and communication policies, a way of recognizing the right to communication would express. However, the demand by which the need for communication as a right is expressed not necessarily wear out the range of possible forms of communication rights nor the ways to address them. 
This paper proposes to test, in the case of Argentina, the arguments supporting the idea that current policies of public communication are based upon the principles of pluralism and democracy, and also it is possible to show a variety of forms of appropriation and use of means under diverse needs. It is essential for communication policies and development of diversity to recognize the multiplicity of symbolic needs and their ways of satisfying them.

Key words: Communication Rights, Communicational Needs, Appropriations and Uses of Radio, Communication and Culture.

\section{Introducción}

El siguiente extracto es de Tzvetan Todorov, extraída del ensayo titulado La vida en común, en el que el filósofo búlgaro despliega sus argumentos con el propósito de responder a la pregunta por lo social y los vínculos entre los individuos en la experiencia colectiva, actualizando la idea del reconocimiento a partir de la dialéctica hegeliana:

"Chateaubriand tiene una necesidad infinita de los otros, pero se preocupa poco por saber si los otros tienen, a su vez, necesidad de él; se cree exento del intercambio social entregando obras al público" (Todorov, 2008: 137).

En una parte del ensayo se detiene en la dimensión simbólica de las prácticas sociales y trata de instalar con esa frase lo que constituye para este trabajo una de las tensiones de la preocupación por la relación entre medios de comunicación y públicos mediada por las necesidades de unos y otros. Chateaubriand es un escritor y político francés del siglo XIX, muy talentoso, pero que no tiene el reconocimiento del gran público al que él le entrega sus obras. Su mayor anhelo es lograr el reconocimiento de un determinado público de la época, pero como sus obras revisten cierto exotismo para el momento, entonces Chateaubriand se frustra porque sus obras están destinadas al ostracismo. El escritor se sitúa en la encrucijada de mantenerse en su torre de marfil y satisfacer su necesidad de creación; o buscar en un intercambio comunicacional con el público los modos y las tematizaciones que los públicos están dispuestos a fijar como objeto de deseo para las necesidades de reflexión intelectual o disfrute.

La intencionalidad que persigue el presente artículo no es auxiliar a Chateaubriand para que logre conseguir que el público reconozca la relevancia de su obra. Lo que se pretende, en primer lugar, es poner luz a las categorías e ideas que permitan a los proyectos políticoculturales de comunicación radiofónica revisar y actualizar los modos y las formas con las que se proponen (a través de sus narrativas y sus estéticas) relacionarse con los públicos. Para alcanzar ese objetivo se puede dar cuenta de una variedad de apuestas teóricas que indagaron en las expectativas y modalidades con las que unos públicos se vinculan con unos medios y unos discursos mediáticos a partir de la comprensión de las necesidades que orientan esa relación.

Una segunda dimensión que se propone este trabajo es la referida a la mediación institucional que opera en el encuentro de la radio con el público. En este aspecto interesa recalar en el 
régimen de verdad que habilita la presencia del Estado en el ordenamiento del campo de los medios de comunicación audiovisuales y en el juego de la opinión a través de un conjunto de políticas públicas que tienen a la comunicación como articulador de lo social.

En el caso de la Argentina, un nuevo escenario fue definido por la Ley $\mathrm{N}^{\circ} 26522$ de Servicios de Comunicación Audiovisual, donde se reconoce un estatus de derecho fundamental a la comunicación ${ }^{1}$ y se sostiene en los principios de la libertad de expresión, pluralidad, diversidad, y atención a grupos en estado de vulnerabilidad ( $c f$. Marino, Mastrini y Becerra, 2010) dando un marco de posibilidad a la emergencia de prácticas comunicacionales que disputan el sentido mercantil de la información y la comunicación.

Situamos el desarrollo del trabajo en la frontera norte de la Argentina $^{2}$, donde la configuración resulta heterogénea en cuanto a su componente sociocultural, ya que conviven en el territorio una gran diversidad de grupos étnicos: criollos, inmigrantes libaneses, españoles, italianos, árabes y grupos indígenas nativos de Sudamérica como wichi, chorote, chané, guaraní, tapiete, toba, chulupí. Algunos de estos grupos indígenas corresponden a poblaciones originarias del territorio. Otras comunidades se han asentado en la zona como consecuencia de migraciones producidas por la Guerra del Chaco de fines del siglo XIX.

Posicionar la mirada en este espacio de lo social no reviste un interés etnográfico, sino que los espacios de frontera constituyen experiencias sociales y humanas donde la diferencia entre los otros que conviven, ponen a prueba la innovación política para gestionar lo colectivo en la diversidad.

Las conceptualizaciones que se abordan en este trabajo constituyen el marco de un análisis sobre las modalidades comunicacionales que se ponen en juego en las prácticas de apropiación del medio radiofónico por parte de dos radios locales del contexto del estudio: La voz indígena (ciudad de Tartagal) y Kaaguazú (comunidad de Yacuy). La primera de las

1 Hablamos de derecho vinculado o relacionado a la comunicación para poner en tensión la denominación utilizada habitualmente derecho a la comunicación o derecho a comunicar, ya que como tal no se encuentra formulada formalmente sino aparece vinculada al derecho a la libertad de opinión y de expresión: "Todo individuo tiene derecho a la libertad de opinión y de expresión; este derecho incluye el de no ser molestado a causa de sus opiniones, el de investigar y recibir informaciones y opiniones, y el de difundirlas, sin limitación de fronteras, por cualquier medio de expresión" (Declaración Universal de Derechos Humanos, artículo 19²). Por su parte, la Ley de Servicios de Comunicación Audiovisual impregna de sentido esta denominación en su artículo 2do que expresa: "La actividad realizada por los servicios de comunicación audiovisual se considera una actividad de interés público, de carácter fundamental para el desarrollo sociocultural de la población por el que se exterioriza el derecho humano inalienable de expresar, recibir, difundir e investigar informaciones, ideas y opiniones" (Ley Nº 26522).

2 En este espacio particular del centro-oeste de América del Sur se presentan rasgos muy característicos en la conformación social, económica, étnica, geográfica y política, el cual fue configurado por un proceso histórico donde fue determinante la racionalidad modernizante y la lógica colonial capitalista como sistemática de apropiación de tierras, explotación intensiva e insustentable de los recursos naturales junto al sometimiento de los pueblos originarios y campesinos mestizos como mano de obra barata para la actividad forestal y los ingenios azucareros. 
experiencias corresponde a una emisora gestionada por una organización sin fines de lucro integrada por referentes de siete etnias originarias. La segunda corresponde a una emisora gestionada por un particular, pero instalada dentro de una comunidad rural indígena. El surgimiento de las dos radios tiene su momento previo a la sanción de la Ley de Servicios de Comunicación Audiovisual en la Argentina y si bien el surgimiento y la presencia de las emisoras expresa una necesidad de los grupos sociales, el proceso de implementación de la ley por parte del gobierno nacional no admitió hasta el momento el reconocimiento legal de ninguna de las dos.

Desde esta perspectiva y con apoyo conceptual en el desarrollo de la teoría de las necesidades de Heller (1996), los motivos que orientan la vinculación de los públicos con los dispositivos comunicacionales podrían entenderse como necesidades sociopolíticas sobre los cuales se montan programas o políticas públicas de comunicación. En este enfoque aparece la idea que todo derecho se sostendría en una carencia expresada en la demanda de un colectivo social y que la política es el arte que asigna y distribuye las formas de atender las necesidades demandadas. Sin embargo, en la praxis política, las demandas expresadas o visibles no agotan la diversidad de formas posibles de demandas sociales ni tampoco las maneras de atenderlas:

"La asignación o atribución [de necesidades] va siempre acompañada de un tipo de recolocación, de redefinición y reagrupación de las diferencias en identidades [...] La distribución de las necesidades es compleja, porque la sociedad necesita distribuir a un tiempo los tipos (clases) de necesidades y lo que las satisface. Lo que las satisface también está tipificado y es abstracto, y las dos abstracciones se relacionan normalmente entre sí” (Heller, 1996: 87).

Se asoma aquí la relevancia por objetivar el arte de gobierno, la manera en que se articulan desde las políticas públicas de comunicación, el reconocimiento por parte del Estado de esa diferencia, de esas singularidades de la comunicación encarnadas en formas de necesidades de ciertos grupos sociales o públicos periféricos y que se cristaliza en las modalidades de apropiación de los dispositivos técnicos y de los discursos mediáticos. También resulta relevante la pregunta por la manera en que los programas de gobierno piensan y asumen los a priori de las modalidades con la que los públicos deberían apropiarse de la comunicación y los bienes culturales para construir el sentido de "lo público". Consecuentemente, cómo en ese proceso de negociación y reconocimiento de la diferencia y la heterogeneidad, se pone en tensión la construcción de la comunidad y la identidad.

En los posicionamientos asumidos en esta dimensión, la acción se orientaría o bien al despliegue hacia la heterogeneidad o la clausura hacia la igualación. Aquí, en este escenario problematizado aparecen entonces un conjunto de operaciones que se despliegan desde espacios de poder para articular el derecho a la comunicación (y las políticas de comunicación) con unas formas de apropiación social y las necesidades que las sustentan.

El presente trabajo se propondrá ensayar preguntas y argumentos en torno a la siguiente presunción: si en el caso de la Argentina las políticas públicas de comunicación 
implementadas por el Estado nacional a partir del año 2009 reconocen como fundamento los principios de pluralidad y democracia que sustenta la Ley de Servicios de Comunicación Audiovisual y diferentes tratados internacionales; si en la observación de las prácticas comunicacionales de ciertos colectivos sociales es posible dar cuenta de una diversidad de modalidades de apropiación de los medios en virtud de una heterogeneidad de experiencias culturales y necesidades simbólicas; resulta entonces fundamental para la implementación de las políticas en comunicación reconocer la diversidad de necesidades simbólicas y de sus formas de satisfacerlas de modo que permitan a los diferentes grupos sociales de la región fortalecer sus estrategias de desarrollo ( $c f$. White, 1992).

\section{EI derecho a la comunicación como atribución de necesidades sociopolíticas de comunicación}

¿Cómo aparece la pregunta por la necesidad en prácticas comunicacionales? En primer lugar, se nos manifiesta desde lo empírico interpelados por ciertas incomodidades inducidas por las formas de apropiación de los dispositivos y discursos mediáticos que se verificaban en determinados grupos sociales en el contexto de intervenciones en el territorio realizadas desde la Carrera de Comunicación Social de la Sede Regional Tartagal de la Universidad Nacional de Salta (Argentina).

Esa incomodidad nos llevó a preguntarnos desde el más puro sentido común por las necesidades que orientaban los modos de apropiación y uso de los dispositivos de comunicación y cómo esas necesidades eran reconocidas por las políticas de comunicación en el marco de la Ley de Servicios de Comunicación Audiovisual, sancionada en el año 2009 en la Argentina.

Ese problema empírico comenzó gradualmente a mudar en problema teórico cuando comenzamos a indagar en tradiciones, textos y autores que ponían en juego la categoría de necesidad en relaciones sociales y prácticas comunicacionales. Pero la pista que nos habilitó a visitar la noción de necesidad se nos develó al releer el Informe MacBride, considerado una declaración de principios para distinguir a los medios de comunicación en su responsabilidad para la construcción de la esfera pública democrática y que aparece nutriendo como fundamento para las normativas y políticas comunicacionales. Este informe, en las recomendaciones relacionadas a los Derechos Humanos, formula:

"Las necesidades de comunicación en una sociedad democrática deberán satisfacerse mediante la extensión de derechos específicos tales como el derecho a ser informado, el derecho a informar, el derecho a la intimidad, el derecho a participar en la comunicación pública, elementos todos estos de un concepto nuevo, el derecho a comunicarse" (MacBride y otros, 1987: 234).

En esta afirmación advertimos que la enumeración de los derechos específicos no agota las posibilidades de invención político-cultural de nuevos derechos que se desprenden 
de procesos de diferenciación y que nutren la construcción de lo común bajo ciertas experiencias de gobierno.

Con un enfoque similar al informe MacBride, el documento conocido con el nombre de "Protocolo 32, anexo al Tratado de Ámsterdam", que también suele citarse como antecedente en la fundamentación de las políticas comunicacionales, advierte que el servicio público de radiodifusión está directamente relacionado con las necesidades democráticas, sociales y culturales ( $c f$. Mastrini, Bizberge y De Charras, 2013).

En estas dos referencias, por citar solo dos, encontramos la noción de necesidad como fundamento de las políticas comunicacionales y de la radiodifusión entendida como servicio público, es decir, que se supondría la preexistencia de requerimientos a suplir o necesidades que se aliviarían o se satisfarían mediante la presencia o uso individual y/o social de los diversos dispositivos o productos comunicacionales. En el caso de la legislación particular de la Argentina (en materia de comunicación la Ley de Servicios de Comunicación Audiovisual) lo formula de la siguiente manera en su artículo $2^{\circ}$ :

"El objeto primordial de la actividad brindada por los servicios regulados en la presente es la promoción de la diversidad y la universalidad en el acceso y la participación, implicando ello igualdad de oportunidades de todos los habitantes de la Nación para acceder a los beneficios de su prestación. En particular, importa la satisfacción de las necesidades de información y comunicación social de las comunidades en que los medios estén instalados y alcanzan en su área de cobertura o prestación” (Ley N²6522, 2009).

La reiterada presencia de la noción de necesidad en la fundamentación del derecho a la comunicación parecería conducirnos a la afirmación de la hipótesis de que existen necesidades individuales y sociales en materia de comunicación. Sin embargo, al profundizar en las discusiones ontológicas y filosóficas en torno a esta idea, se dificulta el acceso a los saberes que se precisan para un estudio sobre los sentidos de las políticas públicas de comunicación. Justamente por las consecuencias que en la praxis política implica la construcción de la categoría de necesidad en el contexto de nuestro interés, es que se vuelve menester clarificar los supuestos que la han configurado para pensar la relación entre gobernantes y gobernados.

Por lo pronto, se evidencia una tensión que emerge en los supuestos teóricos, preliminarmente revisados, para la construcción de la noción: por un lado, una mirada universalista que sustenta la existencia de unas necesidades colectivas; por otro, sistemas teóricos donde las necesidades aparecen como preferencias individuales o particulares. ¿Cuáles son los límites que le reconocemos a estos enfoques? Si nos recostamos en las necesidades colectivas enfrentamos la amenaza latente de la homogeneidad, de la impugnación a la heterogeneidad, luego la coagulación del flujo de la diferencia que conduce a la igualación. En el segundo supuesto, asumir las necesidades desde las preferencias individuales, implicaría pensar en un Estado como articulador de demandas y satisfacciones en términos individuales ( $c f$. Mastrini, Bizberge y De Charras, 2013), agotando el potencial social de lo colectivo. 
En los posicionamientos de los documentos señalados al comienzo, se advierte que los portadores de las necesidades no serían exclusivamente los individuos (postura propia de los enfoques liberales). También la sociedad, como sujeto colectivo "cultural y comunicativo", aparece como portadora de necesidades para, en un caso hipotético, la construcción de una determinada cultura societaria y política, de un determinado orden social. Desde esta perspectiva podría asumirse que la consagración del derecho se produce cuando hay un "reconocimiento de la desigualdad y la exclusión material y simbólica de algún sector de la población" (Mastrini, Bizberge y De Charras, 2013: 13). Sobre estas necesidades (configuradas en y por el complejo de dispositivos de gubernamentalidad) operarían entonces los programas o políticas de comunicación y cultura.

Para comprender estas articulaciones entre la diversidad de demanda y el derecho a la comunicación (en el contexto particular de las radios indígenas en el norte de Argentina) resultó fundamental el reconocimiento efectivo por parte del Estado nacional la legitimidad de esa diversidad, de esa otra manera de "ser" y de "estar" en el mundo reclamado por los pueblos originarios de la Argentina desde mediados del siglo XX y con más fuerza institucional a partir de 1989 con el impulso dado por la firma del Convenio 169 de la Organización Internacional del Trabajo sobre Pueblos Indígenas y Tribales en Países Independientes.

El realce que sobre la diversidad cultural realiza la Ley de Servicios de Comunicación Audiovisual, fue un peldaño más de ese proceso de demanda por el otorgamiento de ciudadanía a las comunidades indígenas de todo el mundo, tal como lo expresa Lizondo (2015) en su estudio sobre la comunicación con identidad y el caso de la radio $\mathrm{La} \mathrm{Voz}$ Indígena. Para los pueblos indígenas, la demanda de ese derecho a la comunicación no es la misma demanda liberal por una opinión libre, por la libertad de expresión, sino que (siguiendo a Lizondo) constituye una herramienta para el resguardo de las formas culturales propias. Esta intencionalidad se explicita en la Declaración de Naciones Unidas sobre los Derechos de los Pueblos Indígenas (ONU, 2007) que en el artículo 16 expresa lo siguiente:

"1. Los pueblos indígenas tienen derecho a establecer sus propios medios de información en sus propios idiomas y a acceder a todos los demás medios de información no indígenas, sin discriminación.

2. Los Estados adoptarán medidas eficaces para asegurar que los medios de información públicos reflejen debidamente la diversidad cultural indígena. Los Estados, sin perjuicio de la obligación de asegurar plenamente la libertad de expresión, deberán alentar a los medios de información privados a reflejar debidamente la diversidad cultural indígena".

El reconocimiento de la diversidad está presente en Ley de Servicios de Comunicación Audiovisual, a partir de la recuperación las Declaraciones y Planes de Acción de las Cumbres Mundiales de la Sociedad de la Información de Ginebra y Túnez de 2003 y 2005. Es a partir de estas recomendaciones (emanadas por la ONU) que se habilitaron políticas comunicacionales con una perspectiva de "diversidades". 
Es necesario advertir que si bien en este trabajo nos hemos dedicado particularmente a la diversidad cultural (a partir de distinciones e identidades de tipo étnicas) la mirada no debe limitarse a ese tipo de diversidad, sino que una verdadera política de la diversidad debe reconocer la multiplicidad de formas de otredades que dialogan y pugnan (con menor o mayor ímpetu) por su reconocimiento y que nos interpelan para que (desde la comunicación) se piensen las herramientas para que la política pueda administrar el flujo de las demandas en su diversidad, comprendiendo la multiplicidad de necesidades que movilizan las demandas de los grupos.

\section{Problematización en torno a la noción de necesidad}

Plantearse interrogantes con relación a la noción de necesidad (para dar cuenta de las prácticas sociales vinculadas al uso de los dispositivos comunicacionales) implica partir del reconocimiento la noción de necesidad como una categoría compleja que requiere de una tarea de recuperación de múltiples abordajes y discusiones analíticas. Es que la idea de la necesidad ha servido como categoría para elucidar aspectos de la acción del sujeto en la vida cotidiana y en la configuración del orden social y económico, ocupando el interés de diferentes disciplinas y enfoques de las humanidades y las ciencias sociales.

Desde estos lugares ha colonizado las prácticas políticas de gobierno para la administración y distribución de bienes y el ejercicio del poder en la modernidad. En nuestro primer abordaje a la problemática, hemos recuperado algunos textos que nos permitieron encolumnar los posicionamientos para darnos una idea de cómo configurar el estado del arte en las discusiones sobre la noción de necesidad, pretensión que no resulta para nada sencilla.

En el contexto contemporáneo, parte de las discusiones en torno a las necesidades pueden ser clasificadas a partir de las posiciones frente a dos dilemas o tensiones: universalismo/ relativismo y subjetividad/objetividad. Por un lado, se sostendrá la existencia de necesidades naturales, verdaderas, objetivas que deben ser cubiertas a todos por igual y de la misma manera. Por otro lado, se sostiene que las necesidades humanas se valoran y configuran en la individualidad del sujeto y que las formas de suplirlas estarían determinadas por un conjunto de preferencias individuales y culturales (Puig, Sabater y Rodríguez, 2012: 2).

Ya el pensamiento sociológico clásico recaló en la noción de necesidad para explicar el cambio y el orden social en la sociedad industrial del siglo XIX. En la propuesta de Durkheim (1967) los seres humanos poseían necesidades de dos tipos: las naturales o primarias y las sociales o secundarias. Siendo que la mayoría de las necesidades son generadas socialmente, el anhelo por mejorar las condiciones individuales y colectivas las vuelve ilimitadas. Para evitar que en la lucha por satisfacer esos apetitos conduzcan a un estado de anomia, se requiere la existencia de una autoridad moral que regule la asignación y distribución.

Entre los autores clásicos, quien más se ha interesado en esta noción ha sido Marx, abordando el concepto con profundidad en los Manuscritos de economía y filosofía (1844) y en La ideología alemana: 
"La necesidad del hombre y el objeto de la necesidad están en correlación: la necesidad se refiere en todo momento a algún objeto material o a una actividad concreta. Los objetos 'hacen existir' las necesidades y a la inversa las necesidades a los objetos. La necesidad y su objeto son 'momentos', 'lados' de un mismo conjunto. Si en vez de analizar un modelo estático analizamos la dinámica de un 'cuerpo social' (en el supuesto de que ese 'cuerpo social' admita una dinámica), entonces la primacía corresponde al momento de la producción: es la producción la que crea nuevas necesidades. En efecto, también la producción que crea nuevas necesidades se encuentra en correlación con las ya presentes: 'La diversa conformación de la vida material depende en cada caso, naturalmente, de las necesidades ya desarrolladas, y tanto la creación como la satisfacción de estas necesidades es de suyo un proceso histórico"” (ápud Heller, 1986: 43).

En El capital, Marx retomará la noción de necesidad para explicar las relaciones sociales en el seno del capitalismo. El capítulo 1 plantea de entrada la categoría de necesidad cuando define a la mercancía y los dos factores constitutivos para comprender el proceso de producción del capital y en la reproducción de la sociedad capitalista ${ }^{3}$.

El estudio de las necesidades en clave del paradigma crítico ha sido retomado en análisis de procesos contemporáneos y que (sin agotar el estado de los abordajes en la temática) sirven de referentes para pensar la categoría. Entre esos textos destacamos La cultura afirmativa y El hombre unidimensional, de Marcuse (2004 y 1964); La génesis ideológica de las necesidades, de Baudrillard (1972); Teoría de las necesidades humanas, de Doyal y Gough (1994); Las necesidades sociales, de Ballester (1999); Teoría de las necesidades en Marx y Una revisión de la teoría de las necesidades, de Heller (1986 y 1996).

Más allá del análisis marxista, la noción de la necesidad también ha recalado en otros enfoques teóricos. Tomando como referencia el estudio realizado por Puig, Sabater y Rodríguez (2012) sobre necesidades humanas, podemos recuperar las conceptualizaciones generales que se elaboraron desde las tradiciones del pensamiento social de procedencia norteamericana. Desde la perspectiva funcionalista, se sostiene una postura relativista de las necesidades (Giddens, 2007). En este sentido, Parsons plantea que el sistema social, ese todo estructurado de partes interdependientes que se orientan a la estabilidad

3 "La mercancía es, en primer lugar, un objeto exterior, una cosa que merced a sus propiedades satisface las necesidades humanas de tipo que fueran. La naturaleza de esas necesidades, el que se originen, por ejemplo, en el estómago o en las fantasías, nada modifica el problema. Tampoco se trata aquí de cómo esa cosa satisface la necesidad humana, de si lo hace directamente, como medio de subsistencia, es decir como objeto de disfrute o a través de un rodeo, como medio de producción" (Marx, 2014: 232). En este texto Marx no profundiza mucho en su análisis; se apoya en Nicholas Barbon para describir cómo opera la necesidad y en una nota al pie lo cita textualmente: "El deseo implica necesidad; es el apetito del espíritu, y tan natural como el hambre al cuerpo [...] la mayor parte [de las cosas] derivan su valor del hecho de satisfacer las necesidades del espíritu" (ibídem). Esta cita nos da una pista de que Marx estaría pensando en dos tipos de necesidades: las del cuerpo y las del espíritu. 
"está compuesto por tres sub-sistemas que se vinculan con unos tipos de necesidades: sistema de la personalidad (necesidades individuales y motivaciones), y el sistema cultural (valores y creencias compartidas), el sistema social (variedad de papeles sociales y normas). [...] Las necesidades son el resultado de la internalización en el sistema de la personalidad (socialización) de las pautas culturales que están institucionalizadas" (ápud Puig, Sabater y Rodríguez, 2012: 14).

Por otro lado, desde la fenomenología (Shütz y Lukmann, 1973; Berger y Luckmann, 1986) y la etnometodología (Garfinkel, 2006 y Goffman, 1981) también se ha problematizado sobre las necesidades y en estos enfoques se las considera como construcciones sociales "a través de interacciones de la vida cotidiana y son subjetivas y dinámicas dependiendo del entorno en una estructura determinada" (Puig, Sabater y Rodríguez, 2012: 4).

En el mismo contexto (y en cierta medida conectada con los dos aportes señalados precedentemente) se encuentra la línea de los estudios sobre "usos y gratificaciones" mediante la cual se esclarecerán las motivaciones que orientan el uso de determinados contenidos de los medios masivos de comunicación por parte de los individuos que conforman las audiencias (McQuail, 1969). El inconveniente de esta línea de estudios es que (al profundizar en procesos psicológicos de la conducta del individuo) no alcanza a aportar elementos para de comprender procesos de apropiación sociopolítica de los aparatos y de los discursos comunicacionales por fuera de los grandes conglomerados de la comunicación.

Donde sí encontramos elementos apropiados a nuestras inquietudes es en la propuesta de Heller (1996) quien sostiene que la necesidad opera en todos los individuos en tanto sujetos sociales. Reconoce que la forma en que ha sido pensada la categoría fue en términos dicotómicos (ya señalada párrafos arriba) y que se requiere superar esa dicotomía, para lo cual propone pensar el complejo de las necesidades en las sociedades modernas con un esquema tripartito que cubre desde la dimensión psicológica, la subjetiva hasta las sociales. Ese esquema se compone por los deseos, las necesidades y las carencias o necesidades sociopolíticas. Si partimos del supuesto de que todo individuo experimenta necesidades, la relación de los elementos de la tríada es expresada de la siguiente manera por la autora:

"El deseo manifiesto (directa o indirectamente) muestra una relación psicológico-emocional y subjetiva con las necesidades, mientras que las carencias (necesidades socio políticas) describen un tipo o clase de necesidad que la sociedad atribuye o asigna a sus miembros (o a alguno de sus miembros) en general. Las necesidades son interpretadas y determinadas de ambas formas" (Heller, 1996: 85).

Si llevamos este esquema al plano de la relación de los individuos con los dispositivos comunicacionales, podríamos ejemplificar de la siguiente manera una hipotética necesidad de esparcimiento como una necesidad general sociopolítica (una carencia). El esparcimiento es una abstracción que abarca todas las formas de distracción del individuo, sin importar de qué manera concrete esa distracción. Pero, siguiendo a Heller, el individuo no expresaría la necesidad de 
esparcimiento, sino una necesidad concreta de salir a dar un paseo, mirar algún programa en la televisión, o escuchar tal programa de radio, etc. Asimismo, muchos deseos se vinculan con necesidades: el deseo que en el paseo me encuentre con tal o cual persona, que disfrute en plenitud el programa, o que en el programa escuche una entrevista que me conmueva.

Ya señalamos en la introducción la dificultad que Heller encuentra en la manera en que se distribuyen socialmente los objetos que satisfacen las necesidades sociopolíticas. Aquí el planteo de la autora recala en las diversas maneras en que se atribuyen y distribuyen las necesidades en la modernidad en contraste con épocas pre-modernas, anteriormente a la modernidad el individuo, en virtud de su cuna heredaba un conjunto de necesidades y las formas de su satisfacción. La modernidad hace estallar esta determinación, dejando al individuo recién nacido desnudo de necesidades, solo con las biológicas. Será entonces el propio devenir del individuo el que irá cargando de necesidades y forjando los objetos de su satisfacción. Esta diversidad cualitativa de las necesidades hace compleja la atribución y la distribución agrupando en el proceso diferencias e identidades.

\section{Apropiaciones sociales y necesidades de la comunicación radiofónica}

Desde los aportes de la sociología de la cultura y los medios de comunicación realizados por Williams (2003, 2011 y 2015) puede pensarse que las prácticas sociales están motivadas por necesidades materiales y simbólicas que los individuos van a tratar de satisfacer para su desarrollo. Según Williams, los modos de satisfacer esas necesidades estarán orientados, por "la materialidad ontológica de la existencia humana y el desarrollo histórico de las formaciones culturales" (ápud Stevenson, 1998: 303), por las formas político-económicas y por las formas tecnológicas. Esta afirmación surge o se desprende de la idea de que todas las prácticas sociales poseen dimensión material y simbólica y que la cultura es la dimensión simbólica de todas las prácticas sociales. En la propuesta de Williams (2015) se distingue, dentro de las prácticas sociales, unas prácticas específicamente significantes o sistemas significantes realizados con cuya denominación va a referirse a las prácticas del lenguaje. Para Williams, estas prácticas específicamente significantes poseen una dimensión material, pero, a diferencia del resto de las prácticas sociales, tanto la dimensión material como la dimensión simbólica sólo tienen la finalidad de significar ${ }^{4}$ (ibídem).

La misma distinción entre la dimensión material y simbólica de las prácticas, Williams la aplicará a las necesidades asignándoles una doble manera de surgimiento: biológico y social. Esta posición también la encontramos en Soper, para quien las necesidades están vinculadas con el fundamento material y con el desarrollo cultural donde las dimensiones de lo biológico y lo social están interrelacionadas (ápud Stevenson, 1998).

4 A partir de esta concepción es que entendemos a la comunicación como una de las prácticas específicamente significantes. Si uno es consecuente con el pensamiento de Williams debemos asumir que esa práctica significante, la comunicación, es constitutiva de la cultura y de la sociedad. Porque si la construcción de los significados en el ámbito de las prácticas se realizan a partir de la discusión, debate y puesta en común, esa construcción de los significados no puede lograrse sin el recurso de la comunicación. 
En la misma línea culturalista encontramos el desarrollo de Stevenson quien, reconociendo las críticas posestructuralistas a la noción de necesidad ${ }^{5}$, realiza una fuerte apuesta por sostener el carácter emancipador de pensar que los seres humanos tienen necesidades para subsistir y prosperar que son satisfechas de diferentes maneras y en diferentes grados por las diversas organizaciones de la sociedad. Stevenson pone su esfuerzo en conciliar la tensión que se expresa entre la perspectiva pluralista y la universalista al considerar la noción de necesidad (ibídem). El punto de partida de la argumentación sostiene que todos los seres humanos experimentamos similares estados físicos y biológicos y compartimos necesidades materiales entre las que se encuentran las formas de comunicación que son, siguiendo la conceptualización de William, a la vez materiales y simbólicas.

Ahora bien, Stevenson supone que no deberíamos derivar las necesidades culturales de las necesidades materiales o biológicas, pues esa lógica esencialista llevaría a agotar la noción de pluralidad y de cultura. Los medios de comunicación contribuyen (de alguna manera) a esa pluralidad de experiencias. Para este esquema conceptual se definen cuatro tipos necesidades que vinculan la comunicación y la cultura en el contexto actual:

“1) la necesidad de conocer el funcionamiento de las culturas expertas; 2) la necesidad de comprender los deseos, las demandas y las interpretaciones que de sus necesidades hacen otros distantes en el tiempo y el espacio; 3) la necesidad de comprendernos como comunidad social, y 4) la necesidad de experiencias humanas de carácter estético y no instrumental" (Stevenson, 1998: 305).

Williams (2015) retoma el análisis de las necesidades y los usos sociales y lo aborda examinando el modo en que se organizan prácticamente en los contextos sociales específicos. Tomando como eje el caso de los productos artísticos y culturales, insistirá que las necesidades están vinculadas a las relaciones de producción (Williams, 2011). En esta obra (al igual que en la tesis propuesta en La larga revolución, 2003) se sostiene que en la organización de las necesidades de carácter material y simbólico operan, de manera determinante, los procesos educativos y de aprendizaje configurados histórica y socialmente.

Para Williams (2015), la organización del sistema educativo en la Inglaterra del siglo XIX (cuando ubica la mayor transformación) tuvo un sentido clasista, y los compartimentos resultantes obedecían a las necesidades de las diversas clases sociales y funciones que la sociedad moderna requería para su evolución. Para Williams, las necesidades son o deben ser organizadas por el Estado pues su orientación debe servir para la construcción de la organización social. Es la necesidad de una entidad mayor, abstracta, la que orienta la práctica. No sería la satisfacción de una pulsión colectiva o individual (ibídem).

5 Desde el posestructuralismo se realiza un cuestionamiento a la noción de necesidad por considerarla un concepto inútil por cuanto esa noción supone un sujeto homogéneo desconociendo la diferencia y pluralidad de experiencias de vida. Si bien le reconoce cierta validez a esta posición pluralista, Stevenson va a plantear en primer lugar que negar las necesidades llevaría a anular todos los esfuerzos de una teoría crítica de las necesidades. 
Con estos elementos comenzamos a observar lo que sucede en las prácticas de la comunicación radiofónica. Lo primero que advertimos es que el hecho tecnológico denominado "radio" (compuesto por unos aparatos mediante los cuales la sociedad va a poder enviar-recibir información en soporte de sonido de manera masiva) encontrará su reconocimiento como medio de comunicación social en los usos sociales, es decir, en el momento en que incorpora unas temáticas (contenido) y unas modalidades discursivas (formas) que son apropiadas por las audiencias como relevantes para la vida cotidiana y por lo tanto satisfacen necesidades de carácter social y cultural. Tal como expresa Williams, la radio es una forma heredada de otras prácticas sociales y culturales. En tal sentido, los contenidos radiofónicos son significativos no solo por ser difundidos en la radio, sino porque esas temáticas y modalidades tienen una presencia social y cultural previa a su incorporación en el flujo discursivo que proponen las emisoras.

No atender al planteo anterior podría inducirnos la idea que la insistente presencia del aparato de radio en la vida de las personas desde hace cien años fue exclusivamente obra de empresarios con ánimo de lucro. Sostener esta hipótesis implicaría un determinismo tecnológico o económico de los procesos de la cultura masiva y obturaría los esfuerzos de los proyectos comunicacionales para alcanzar la aceptación de su público. Además, se disolvería el sentido a la cuantiosa experiencia radiofónica (que desde lo alternativo y lo comunitario) han podido aportar a la multiplicación de diversidades de tematizaciones y modalidades estéticas de la comunicación.

Para comprender el desarrollo de surgimiento y consolidación de la presencia social-cultural de la radio, Williams plantea que se requiere prestar atención a un sistema comunicacional más amplio anterior a los desarrollos de la radiofonía (ibídem). En la misma línea, la respuesta que Martín-Barbero (2010: 195) encuentra (en esa presencia de la radio en la vida cotidiana de nuestra sociedad) remite claramente "al proceso que 'conecta' la radio con una larga y ancha tradición de expresiones de la cultura popular" a unos discursos con una fuerte existencia social previa.

Siguiendo esta argumentación de Martín-Barbero, sería posible pensar la conversación como antecedente del formato radiofónico magazine; el rumor social y la noticia gráfica como antecedente de los formatos informativos y noticiosos; las discusiones en los bares o clubes sociales como antecedente de los formatos de opinión y discusión; el fútbol, el boxeo, las carreras cuadreras, las riñas de gallos como antecedentes las transmisiones de espectáculo de destreza física; los juegos de mesa familiares y las quermeses como antecedentes a los programas de juegos y entretenimientos tipo preguntas y respuestas; de igual modo que la música, los eventos populares o la publicidad.

Tomar como punto de partida la cultura nos facilita el desplazamiento y la conexión con espacios sociales donde se facilita la relación entre unos sujetos (con pertenencias históricas y sociales) y unos discursos radiofónicos cuyas condiciones de producción también son situadas históricamente. Esa relación (recepción/consumo) se da en la vida cotidiana y es considerada como momento privilegiado de producción de significaciones. De esta manera, la conceptualización de la cultura se definirá como aspecto activo de la vida en sociedad y en el consumo de los medios de comunicación. 
Desde esta perspectiva, el receptor no aparece aislado y alienado en la pasividad, sino que se sostiene en la red de significados y sentidos que va tejiendo en su devenir. La idea del receptor pasivo le deja el lugar al actor-productor, productividad que emerge en las modalidades de decodificación (Hall, 1980) y en las apropiaciones y usos que hacen los públicos de las tecnologías y los discursos radiofónicos. Este fenómeno admite la coexistencia de modalidades diferenciadas de apropiación y uso en virtud de la diversidad de maneras en que se expresan las necesidades comunicacionales y simbólicas de los diversos públicos, así como de las modalidades de acceso a los aparatos y discursos radiofónicos.

Dar cuenta de las modalidades de uso y la disponibilidad tecnológica nos permite conectar las condiciones de recepción y producción con las necesidades simbólicas que son convocadas por las radios locales. Aquí se asoma la noción de apropiación en la que Martín-Barbero recala para describir las relaciones y sentidos que unen a los sujetos con bienes o contenidos culturales que consumen. Uno se apropia de lo que le resulta propio, apropiado y en esa relación intervienen factores como hábitos, competencias culturales, modalidades que responderían a posiciones sociales y culturales (Martín-Barbero, 1991: 15).

Un último planteamiento que nos pareció relevante convocar (antes de describir las experiencias radiofónicas) es que los procesos de apropiación no son lineales, universales ni deterministas. En Williams (2015) aparece la idea de que las necesidades asociadas a los medios de comunicación pueden leerse como causas o consecuencias de un complejo proceso de transformaciones sociales, económicas, culturales, tecnológicas, etcétera, que en el devenir histórico se van influyendo mutuamente. De aquí que (para comprender la dinámica de las necesidades, apropiaciones y usos sociales actuales de la radio) uno debe distanciarse del objeto específico y reconocer los movimientos o mutaciones que se configuran en un espacio de articulación más amplio donde entran en juego las narrativas y dispositivos transmediales y multimediales.

Aquí arriesgamos una lista tentativa y para nada definitiva de transformaciones que requerirían tenerse en cuenta para pensar las necesidades comunicacionales en el contexto actual:

- "Las de las formas de uso de las tecnologías de la comunicación: la recepción (información, entretenimiento, educación, compañía, conversación), los dispositivos de control o la organización social, la creación artística, la identidad cultural, las relaciones sociales.

- La de las necesidades de los individuos que son creadas por la industria pero que también se derivan del desarrollo de histórico de la sociedad.

- Las de las formas del mundo del trabajo: ampliación del tiempo y del espacio donde se desarrolla la actividad laboral (sobre todo el trabajo intelectual y cognitivo) a partir de la incorporación de las TIC.

- Las del aprovechamiento del tiempo de ocio y las nuevas formas del entretenimiento individual y colectivo.

- Las de las formas de intercambio culturales y simbólico-narrativas: el chat, el tweet, la producción colaborativa, los relatos transmediales, realidad virtual, video juegos multijugador. Aquí resulta esclarecedor el planteo de Berardi de 
la crisis en la transmisión cultural entre las generaciones alfabético-críticas y las post-alfabéticas" (Berardi, 2007: 25).

- "La de las tecnologías (que se ajustarán más o menos a las necesidades), el crecimiento y multiplicación de las tecnologías de distribución 'persona a persona', la reinvención de las tecnologías del broadcasting a partir del surgimiento de nuevas redes de distribución de contenidos (especialmente Internet), y las combinaciones entre ambas. Tal como lo plantea Williams el proceso de apropiaciones y usos sociales de las tecnologías de la comunicación es complejo y en casos, como el de la televisión, la ciencia y la industria buscan el desarrollo de una nueva forma de tecnología que se ajuste a las formas sociales o a necesidades sociopolíticas; otras veces, como en el caso de la radio, "un conjunto de artefactos técnicos aislados se convirtió en tecnología aplicada y luego en tecnología social” (Williams, 2011: 38).

La lista no resulta para nada exhaustiva, pero en el resultado de las articulaciones de estos fenómenos se advierten la multiplicidad de preferencias y de disposiciones en relación a los medios. A partir de diversas investigaciones sobre usos tecnológicos y consumos culturales en nuestro país es posible advertir en el uso extendido del binomio smartphone + Internet una novedosa modalidad de apropiación de los públicos en el terreno de lo popular para comunicar $\mathrm{u}$ informarse. Aquí es posible dar cuenta de prácticas que responden a necesidades simbólicas anteriores a las tecnologías electrónicas: la conversación, la narración, la música, el afecto, la organización política, el espectáculo.

Cada una de estas necesidades posee una pluralidad de maneras de ser satisfechas que hay que indagar previamente para pensar las políticas públicas de comunicación, pero sin dudas la radio las ha sabido comprender y resolver de una manera apropiada para sectores populares. Entonces se debe agudizar la mirada de los rituales, de los lugares sociales y culturales que ocupan las diferentes tecnologías y narrativas (además de las específicamente radiofónicas) para comprender y dar cuenta de las necesidades simbólicas que orientan las disposiciones (preferencias y usos) de los medios (con sus tecnologías y narrativas) por parte de los diversos públicos.

\section{Necesidades y apropiaciones de la radio en comunidades y colectivos sociales del norte de Argentina}

Hasta aquí (y con los elementos que hemos desarrollado) podemos coincidir que, así como el derecho a la comunicación no puede ser reducido a su formato jurídico (para dar cuenta de la diversidad de modos de reclamo), la teoría de la necesidad también solicita ser pensada más allá de la formulación psicoligisista o la subsunción (alienación) del individuo a una especie universal abstracta que es la sociedad. Seguramente encontramos en cada uno de nuestros países programas de gobierno en los que se afirme que se atienden necesidades sociales y se recurre a este universal para explicar por qué se gestiona en tal o cual sentido. Pero esa discusión no tiene sentido en esta parte, ya que no aporta demasiado al análisis que nos convoca. 
Lo que interesa es como aparecen las necesidades diferenciales: lo que reclama la atención es ver como parte de ese abstracto (que es lo social) materializa singularidades que libera fuerzas creadoras e inventivas en las comunidades concretas, en los grupos humanos concretos, que se apropian de la comunicación y ponen en juego prácticas de la crítica. Ese todo homogéneo y vacío de lo social en realidad nunca va a cubrir y agotar las formas en que el derecho a la comunicación se reclama, sino a partir de las formas en que los grupos se apropian de la comunicación.

Son dos experiencias las que nos interpelan para pensar el modo de asignación de derechos a partir del reconocimiento de esa diversidad. El desarrollo de las experiencias no pretende una precisión en el análisis, sino que intenta dar cuenta de prácticas y otras formas de reclamar o de acceder al derecho a la comunicación.

El caso $^{6}$ de la radio Kaaguazú, de comunidad de Yacuy ${ }^{7}$, constituye una emisora de gestión privada y unipersonal que está instalada en el centro de la comunidad. La primera singularidad de este medio es la apuesta que realiza el propietario y gestor de la radio por la instalación de un medio de comunicación en un contexto comunitario. Aquí es relevante el proyecto político comunicacional de la emisora, cuya intencionalidad se esmera por intervenir en el entramado social de la comunidad dinamizando, a través de la oferta comunicacional, procesos de subjetivación del sentido comunitario.

Para materializar ese cometido, la emisora establece articulaciones con las instituciones y grupos sociales de la comunidad para que se produzcan contenidos radiales. De este modo, la radio ha prestado espacios de programación a la Biblioteca Popular, al Centro de Actividades Juveniles, a organizaciones de apoyo técnico y a la Iglesia Evangélica que está instalada en el poblado. La programación se completa con programas producidos por otras radios, como el caso de los boletines informativos de Radio Nacional o programas de radios evangélicas.

Las indagaciones realizadas por el equipo de extensión sobre el público de la radio Kaaguazú se ocuparon de tres tópicos: el lugar social y cultural de la radio en la vida cotidiana de la comunidad; el dispositivo tecnológico que facilita la escucha; las orientaciones de la audiencia en la elección de contenidos. Sobre el primer tópico, los resultados coinciden de algún modo con las conclusiones a las que arribaron diversas investigaciones como

6 Los datos presentados en este caso fueron relevados por el grupo de trabajo del Proyecto de Extensión Universitaria de la Universidad Nacional de Salta (Sede Regional Tartagal) denominado "Misiareta iñe: la voz de los chicos". El equipo estuvo conformado por las estudiantes Noelia Navarrete, Claudia Eslava y Diana Ripalda con la dirección del docente Emiliano Venier. Para dar cuenta del caso se sistematizó la observación participante realizada durante 12 meses de intervención en el apoyo a un programa radial realizado por jóvenes de la comunidad y se complementó con la realización de entrevistas semiestructuradas a miembros de la audiencia de la radio Kaaguazú en las que se indagó en las modalidades de uso y consumo de la radio.

7 Yacuy es una comunidad de la etnia guaraní, integrada por aproximadamente 2500 personas. Es una comunidad periurbana que se encuentra en la zona rural del ámbito de la ciudad de Tartagal ubicada al norte de la República Argentina, a 55 Kilómetros de la frontera con el Estado Plurinacional de Bolivia. 
las de Mata (1988) y Winocur (2002), donde la presencia social y cultural de la radio Kaaguazú en la comunidad de Yacuy se verifica en diversos rituales en la vida cotidiana de los habitantes, tanto en el hogar (tareas de la casa, estudio, descanso) como en las labores agrícolas. La práctica de la escucha tiene dos modalidades: individual y colectiva.

Para describir las modalidades de escucha debemos recurrir al segundo de los tópicos relevados, el de los dispositivos. En el caso de la escucha individual, se realiza mediante el teléfono celular: un dispositivo del que los miembros de la comunidad se han apropiado tanto para la comunicación oral como para la escrita. La escucha compartida es la más habitual en la comunidad, pues la radio con parlante está ubicada en la sala de la casa (en general con un único ambiente) en el patio, o en el espacio laboral.

Con relación al tercer tópico relevado, los programas que tienen una mayor presencia en los intercambios con los miembros de la comunidad son los que transmiten música popular del ámbito rural, música y temas cristianos, o información de actualidad o de interés especial agrícola. En la mención de los entrevistados se destacó el programa "Nuestra tierra, nuestro canto", que se trata de una propuesta de contenidos vinculados al mundo rural, generando un reconocimiento de la principal actividad económica de los miembros de la comunidad. El programa aborda temas relacionados a las diversas labores agrícolas animándola con música rural que de alguna manera representa a esa actividad y responde a los gustos de la audiencia.

Aquí la radio asume un doble aporte a las necesidades de su público de compañía o entretenimiento y de información (general y técnica específica de la actividad agrícola minifundista), respondiendo a la necesidad que Stevenson define como la necesidad de conocer el funcionamiento de las culturas expertas, la necesidad de comprendernos como comunidad social y la necesidad de experiencias humanas de carácter estético y no instrumental.

Otra temática que conserva el reconocimiento del público de la radio son los programas realizados por los miembros el grupo religioso cristiano-evangélico que forma parte de la comunidad. Gran parte de los habitantes de Yacuy profesan la fe cristiana participando de los rituales evangélicos. De esta manera el mensaje religioso se expande en el tiempo y en el espacio con la radio en los hogares y en el trabajo. Otro programa vinculado a la necesidad de comprenderse como comunidad social-espiritual, es el programa "Misiareta iñe" que realizaba un grupo de jóvenes de la comunidad en el que se generaban intercambios sobre las problemáticas propias de este grupo etario.

Para dar respuesta a las necesidades de contenidos informativos de actualidad, la emisora retransmite los boletines informativos de la emisora estatal Radio Nacional que emite contenidos desde la ciudad de Buenos Aires. De esta manera, la radio se constituye un anudador de la vida cotidiana comunitaria con la racionalidad técnica de la sociedad globalizada.

El caso de la radio comunitaria La voz indígena resulta una experiencia que ha sido objeto de estudio de numerosos trabajos, entre ellos el de Lizondo (2015), quien da cuenta de una práctica singular de una emisora comunitaria con identidad indígena, cuya gestión la 
realizan referentes de las siete etnias de pueblos originarios de Sudamérica presentes en la localidad de Tartagal. Esta diversidad tan particular es reflejada en la sonoridad de la radio al momento que los programas son realizados en las lenguas de cada una de las etnias. $L a$ voz indígena es una emisora que articula el espacio urbano con el rural, irrumpiendo en el espectro radioeléctrico de la ciudad con sonidos, voces y cosmovisiones.

Las tematizaciones que incorpora la radio en sus contenidos son elementos consistentes con el proyecto político institucional de la radio. En tal sentido, Lizondo señala como características el abordaje sistemático de temas como las luchas por la tenencia de la tierra (mediante spots y radioteatros), la visibilización de la diversidad de grupos culturales con sus prácticas materiales y simbólicas ${ }^{8}$, el acompañamiento en los conflictos o demandas de las comunidades indígenas y el reconocimiento de las particularidades de esa diversidad cultural en los elementos estéticos transportados por los mensajes y las texturas sonoras.

Además de formar parte del proyecto de la emisora, esas tematizaciones y musicalidades son característicos de los grupos indígenas que forman parte del público. La radio se propone ser una imagen especular de las formas de comunicación y de las tematizaciones de su público. En ese reconocimiento, se funda la vinculación del medio con la audiencia y la apuesta política que los otros grupos culturales comprendan la diferencia.

\section{Conclusiones}

Dentro de la racionalidad occidental, que haya un poder que quiera gobernar a la población solo es pensable, posible, a partir de que ese poder reconozca y atienda las necesidades de la población. En la relación entre gobernados y gobernantes hay una serie de demandas de felicidad social que se ponen en juego en esa articulación ${ }^{9}$. Es en función de la satisfacción de necesidades que se habilita la posibilidad del gobierno de intervenir sobre los gobernados, de conducir la población. Pero, advirtiendo como se desarrolla la noción de necesidad desde los posicionamientos señalados en este trabajo, es menester de desmontar la idea de que el reconocimiento de una necesidad sociopolítica viene a completar una ausencia para el conjunto de la población.

Se requiere cuestionar las teorías de las necesidades porque imponen límites al remitirnos a sujetos preestablecidos, a un todo social, o a un sujeto psicológico que se aliena a los deseos conducidos por el mercado o la ideología. Una teoría de las necesidades vinculadas a la comunicación debe abrir el horizonte de la heterogeneidad y no clausurarlo.

8 Diversidad que no solo se reduce a la diversidad étnica sino que la programación de la radio incorpora programas de organizaciones políticas, colectivos por la diversidad sexual, organismos públicos de salud o educativos.

9 En este punto nos parece importante reseñar el proceso desarrollado por Foucault (2006: 296) del paso de la razón del Estado (como la felicidad del mismo Estado) hacia la razón de Estado como la felicidad de la población: forma propia del liberalismo que aparece como antecedente de lo que aparecerá como la cuestión de la relación entre gobierno y felicidad en el neoliberalismo y quizá más allá de él. 
En Argentina, la Ley 26.522 constituyó un caso tomado como ejemplo de la regulación de las comunicaciones audiovisuales. La ley contribuyó y dio sostén a un movimiento social, político y cultural para potenciar los esfuerzos hacia la pluralidad de voces en la radio y la televisión bajo una ontología de la inclusión. El sentido con que se materializó esa política en la aplicación de los programas de gobierno parece haber encontrado límites que obturaron la consolidación e institucionalización atentas a que no lograron articular una defensa contra la arremetida de los grupos mediáticos concentrados.

Esto podría obedecer a que, en la consideración o atribución de este nuevo derecho cultural como el de la comunicación, se respondió a una lógica de la igualación, de la universalización por sobre la lógica del reconocimiento de las diversidades. Parecería que los esfuerzos de una política por más ciudadanías y derechos que operó bajo la lógica de la inclusión se encaminaron en igualar allí donde no había identidades que se concilien y ese compromiso no permitió advertir la multiplicidad de las experiencias y la demanda diferencial.

Si es correcto esa primera conclusión, no se trataría de que es macro-sujeto universal que es el Estado deba reconocer a cada uno como iguales y asigne y distribuya unos derechos. La política de la inclusión bajo la lógica de lo igual parece encontrar sus límites: el argumento de un Estado que incluye porque es el que otorga soberanamente los derechos a todos los súbditos por igual se agota en sus esfuerzos. Hay actores sociales que con sus prácticas y sus modos de existencias están impugnando y corriendo las fronteras a partir de las cuales la ciencia y el Estado los piensa como sujetos sociales y como sujetos la comunicación.

Lo que se le pide al Estado hoy es que incorpore una política de reconocimiento bajo la lógica de la diversidad de formas de existencia; que se ubique en el núcleo del complejo proceso de articulación entre la multiplicidad de fuerzas culturales y políticas para pensarlas y estimularlas a que desplieguen al máximo su potencial de creación y diferencia. El derecho a la comunicación no es un derecho como los otros porque la vida en común en un contexto de diversidad se basa en las tensiones y relaciones de poder entre las fuerzas culturales y políticas, y esas tensiones y relaciones de poder se dirimen en las disputas por el sentido del orden social, de las formas de pensar lo social, lo cultural y lo político; y las disputas de sentido requieren del despliegue de las técnicas, dispositivos, estrategias y astucias de la comunicación.

Los miembros de la comunidad de Yacuy y los de La voz indígena no reclaman la comunicación de la misma manera que lo hacen las corporaciones mediáticas, los colectivos por la diversidad sexual, o la opinión pública ilustrada. Tampoco organizan sus reclamos con el modo en que suele organizarse para reclamar derechos la ciudadanía de las democracias liberales. Tal vez la demanda de estos derechos en estos sujetos no sea una demanda estridente, no sea una demanda que se formule bajo, o solamente bajo consignas, sino que tal vez lo más interesante en las luchas por estos derechos sea la necesidad de potenciar el componente emancipatorio que hay en las prácticas que sostiene ese singular ejercicio de la comunicación. Más aún, incluso el reconocimiento del derecho a la comunicación podría pensarse no como algo que se accede desde un no derecho absoluto. 
La comunicación no como algo que las poblaciones no tienen y de repente el Estado se la concede como derecho. Al contrario, la comunicación está (y estuvo) constantemente presente articulando las distintas prácticas de los grupos sociales y del gobierno.

El derecho a la comunicación se puede expresar en lo jurídico por la afirmación clara del reconocimiento de ciertos derechos, pero no se pasa del no derecho al derecho, sino que siempre está presente bajo las formas de resistencia, la impugnación de ciertas categorías a partir de las cuales se quiere gobernar. Y eso también forma parte del derecho a la comunicación. El no querer ser gobernado en estos términos (de esta manera, por estas personas) el no querer ser hablado de este modo, el no querer ser mostrado de este modo, el no tener que ser organizado desde estas categorías de lo social. Por eso no es que el Estado viene a reconocer un derecho que los grupos sociales no tenían, sino que aparece, en ese reconocimiento de derecho que el Estado argentino ha otorgado mediante la LSCA, la ampliación de derechos fundamentalmente como una herramienta más que se pone al servicio de ciertas luchas emancipatorias de grupos sometidos.

Pero también nos queda en este análisis que la afirmación formal del derecho a la comunicación no es el final de la lucha, ya que su compromiso se agotará si no contribuye a brindar herramientas para continuar una contienda que siempre estuvo presente en la relación entre gobernados y gobernantes, incluso antes de las formas burguesas de pensar la libertad como libertad individual y ciudadana, y que remite a las formas en que se piensa y se expresa el lugar del dominado. Aquí emerge la dimensión política de la comunicación y la verdadera apuesta política del derecho a la comunicación, en tanto permite configurar el espacio en el que se despliegan los argumentos para discutir los lugares que ocupan gobernados y gobernantes, así como los saberes que permiten pensar y legitimar esos lugares.

La cuestión es que, así como el derecho a la comunicación no puede ser reducido a su formato jurídico, la teoría de la necesidad necesita ser pensada más allá de la formulación psicologista o la subsunción a una especie universal que es la sociedad. Tal vez en los programas de gobierno se exprese que se atienden necesidades sociales y se recurre a este universal para explicar por qué se actúa en tal o cual sentido.

\section{Fuentes consultadas}

Ballester, L. (1999). Las necesidades sociales. Barcelona: Síntesis.

Baudrillard, J. (1972). La génesis ideológica de las necesidades. Barcelona: Anagrama.

Berardi, F. (2007). Generación Post-Alfa: patologías e imaginarios en el semiocapitalismo. Buenos Aires: Tinta Limón Ediciones.

Berger, P. y Luckmann, T. (1986). La construcción social de la realidad. Buenos Aires: Amorrortu 
"Declaración de las Naciones Unidas sobre los Derechos de los Pueblos Indígenas" (2007, septiembre 13). Asamblea General de la ONU.

"Declaración Universal de Derechos Humanos" (1948, diciembre 10). Asamblea General de la ONU. Resolución 217 A (III).

Doyal, L. y Gough, I. (1994). Teoría de las necesidades humanas. Madrid: Icaria-FUHEM.

Durkheim, E. (1967). De la división del trabajo social. Buenos Aires: Editorial Schapire.

Foucault, M. (2008). Seguridad, territorio y población. Curso en el Collège de France (1977-1978). Madrid: Akal.

Garfinkel, H. (2006). Estudios en etnometodología. México D. F.: Anthropos Editorial.

Giddens, A. (2007). Sociología. Madrid: Alianza Editorial.

Goffman, E. (1981). La presentación de la persona en la vida cotidiana. Buenos Aires: Amorrortu Editores.

Hall, S. (1980). "Encoding / decoding". En Centre for Contemporary Cultural Studies (ed.). Culture, Media, Language: Working Papers in Cultural Studies, 1972-79. Londres: Hutchinson.

\section{Heller, A.}

_(1996). Una revisión de la teoría de las necesidades. Barcelona: Editorial Paidós. _(1986). La teoría de las necesidades según Marx. Barcelona: Ediciones Península.

Heller, A. y Fehér, F. (1995). Biopolítica. La modernidad y la liberación del cuerpo. Barcelona: Ediciones Península.

Katz, E. y otros (1985). “Usos y gratificaciones de la comunicación de masas”. En De Moragas, M. (ed.). Sociología de la comunicación de masas [vol. II]. Barcelona: Gustavo Gili.

Lazarsfeld, P. y Merton, R. (1986). “Comunicación de masas, gustos populares y acción social organizada”. En De Moragas, M. (ed.). Sociología de la comunicación de masas [vol. II]. Barcelona: Gustavo Gili.

“Ley No 26.522" (2009, octubre 10). Boletín Oficial de la República Argentina. Buenos Aires.

Lizondo, L. (2015). Comunicación con identidad o comunicación Comunitaria. El caso de la fm "La voz indígena" [tesis de maestría]. Universidad Nacional de La Plata, Facultad de Periodismo y Comunicación Social. Extraída el 26/IV/2016 desde http://sedici.unlp.edu.ar/bitstream/handle/10915/50419/Documento_completo. pdf? sequence $=3$ 
MacBride, S. y otros (1987). Un solo mundo, voces múltiples. Comunicación e información en nuestro tiempo. México D. F.: Fondo de Cultura Económica.

\section{Marcuse, $\mathbf{H}$.}

_(2004). La cultura afirmativa. Buenos Aires: El cuenco de plata.

_(1964). El hombre unidimensional. Barcelona: Ariel.

Marino, S.; Mastrini, G. y Becerra, M. (2010). "El proceso de regulación democrática de la comunicación en Argentina”. Oficios Terrestres núm. 25. Extraída el 3/V/2016 desde http://sedici.unlp.edu.ar/bitstream/handle/10915/45366/Documento_completo_. pdf? sequence $=1$

\section{Martín-Barbero, J.}

_(2010). De los medios a las mediaciones. Comunicación, cultura y hegemonía. México D. F.: Anthropos.

_(1991). "Recepción, uso de medios y consumo cultural". Revista Diálogos de la Comunicación, num. 30.

Marx, K.

_(2014). Antología. Buenos Aires: Siglo XXI editores.

_(1975). Manuscritos económicos-filosóficos de 1844. Barcelona: Grijalbo.

Mastrini, G.; Bizberge, A. y De Charras, D. (eds.) (2013). Las políticas de comunicación en el siglo XXI. Buenos Aires: La Crujía Ediciones.

Mata, M. (1988). "Radio y públicos populares". Diálogos de la comunicación, num. 19. Extraída el 3/V/2016 desde http://dialogosfelafacs.net/wp-content/uploads/2015/19/19revista-dialogos-radios-y-publicos-populares.pdf

McQuail, D. (1969). Sociología de los medios masivos de comunicación. Paidós: Buenos Aires.

Parsons, T. (1999). El sistema social. Madrid: Alianza.

Puig, M.; Sabater, P. y Rodríguez, N. (2012). "Necesidades humanas: evolución del concepto según la perspectiva social”. En Aposta. Revista de Ciencias Sociales, núm. 54. Extraía el 26/IV/2016 desde http://www.apostadigital.com/revistav3/hemeroteca/ monpuigllob.pdf

Schütz, A. y Luckmann, T. (1973). Las estructuras del mundo de la vida. Buenos Aires: Amorrortu Editores.

Stevenson, N. (1998). Culturas mediáticas. Teoría social y comunicación masiva. Buenos Aires: Amorrortu Editores. 
Todorov, T. (2008). La vida en común. Ensayo de antropología general. Buenos Aires: Taurus.

Tratado de Amsterdam (1997). Oficina de Publicaciones de Comunidades Europeas, Bélgica.

White, R. (1992). "Análisis cultural en la comunicación para el desarrollo. El rol de la dramaturgia cultural en la creación de una esfera pública". Revista Diálogos de la Comunicación, núm. 34, pp. 42-57.

\section{Williams, R.}

_(2015). Sociología de la cultura. Buenos Aires: Paidós.

_(2011). Televisión: tecnología y forma cultural. Buenos Aires: Paidós.

_(2003). La larga revolución. Buenos Aires: Nueva Visión.

Winocur, R. (2002). Ciudadanos mediáticos. La construcción de los públicos en la radio. Barcelona: Gedisa. 